\title{
The Rationale for Fundamental Research in Space Biology: Introduction and Background
}

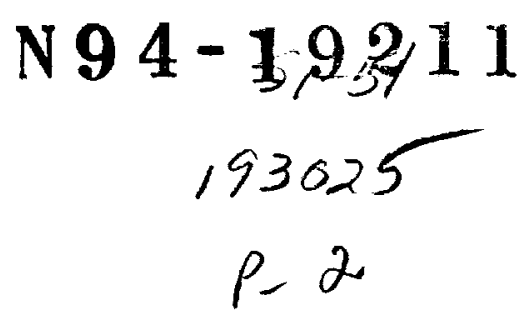

\author{
ROBERT W. KRAUSS \\ NASA Headquarters, Washington, DC 20546 \\ Thora W. Halstead \\ NASA Headquarters, Washington, DC 20546
}

P 2

\section{ABSTRACT}

With the construction of Space Station Freedom, NASA will have available a new platform for experiments in space that promises many advantages over those already flown. Biologists are poised to take advantage of the greater space, the increased power, and especially the long duration of the station for a cascade of innovative experiments in fundamental science that are long overdue. The unique space environment will provide new dimensions for approaching some of the most challenging problems still facing modern biology. Solutions to basic questions about living systems, which may now be grown through many generations in space, will not only explain abnormalities already observed there, but will add to our understanding of how life functions on Earth. Much will be learned about evolution that has built us the way we are, but also about what it has in store for the Earth's species in the future. NASA must not lose this opportunity to contribute to the welfare of the peoples of the Earth while at the same time create knowledge that will enable human exploration of space in the decades ahead.

\section{INTRODUCTION AND BACKGROUND}

During 1991 NASA was visited by a thorough review of its activities by the "Augustine Committee" appointed by President Bush. The committee, comprised of leaders in the aerospace industry and of persons bringing long histories of interaction with NASA, recommended some small changes in organization and directions but reinforced the view that NASA's role in the next century ought to be a major one. NASA should be committed to continue and expand its exploration of space away from Earth and also to strengthen its observation and remote monitoring of Earth itself. All that NASA could hope to do would be part of one mission or the other. With that the simplicity ended and NASA's Divisions and Centers began to reexamine their roles and organization to mesh the two missions and realign many programs already under way.

Life Sciences have shared in the general introspections. Where does space biology belong? Is it just part of medicine, or vice versa? What are the governing forces that determine the nature of the research that it funds? What can it contribute, and even, what is Space Biology?

We are not going to attempt to answer these questions, but rather examine what biological science is attempting to achieve here on Earth as well as in space. Hopefully, enough light can be generated from these papers to help NASA and the scientific community take a fresh look at biology to better plot its strategy from a different perspective. No other science is in a better position to benefit from a continuance of space platforms on which experiments can be performed, and no other science is as likely to make discoveries that will more promptly contribute to the physical and intellectual welfare of humankind. However, at this junction neither biologists, the public, NASA, or Congress are awake to biology's full potential in space in spite of over 30 years of Man's presence there. Historians in the future will surely reflect on the reasons why biology was so slow to exploit the special conditions of spaceflight to the advantage of all.

Perhaps a few words of background will set the stage for the papers that are to follow. First, it is useful to be clear in the definition of "science." Science is directed toward finding and organizing facts or data into a body of knowledge so that a given phenomenon is completely known or understood. The quest for understanding the phenomenon called life is far from over. There is now only incomplete knowledge in spite of the dramatic achievements of biologists in the last half century. Furthermore, those achievements have been characterized by a purity of purpose that is not obscured by the requirement that practical benefit must come promptly.

Second, it is well to recall that useful new technologies have flowed so consistently from basic studies that seek knowledge for its own sake that society takes those contributions for granted. That there is a role for applied research is undeniable, but even applied science advances faster when it is done in the context of sound basic facts and principles. It is therefore imperative that NASA support a basic life 


\section{HALSTEAD AND KRAUSS}

sciences program, as it does with physics and astronomy, to accompany an applied program in space medicine, which is essential to enable astronauts to survive and function in space for extended periods. The two programs can thrive on the synergism that is predicted upon the recognition of clear priorities for each.

Third, some appreciation of the way biology works is necessary. Although biology employs math, physics, and chemistry as its tools to elucidate living systems, the very great complexity of life and the difficulty of segregating variables in even the most focused experiment dictates a somewhat different methodology for research. Work usually begins with an exploration phase looking at performance and function, followed by an analytical phase to identify control mechanisms by repeatedly probing with carefully crafted experiments. It is initially an inductive science with broad principles evolving from a multitude of what often may appear to be unrelated experiments. Testing of principles and premises through deductive reasoning properly integrated and validated then matures into knowledge.

If it is expected to yield sound knowledge of enduring value biology requires time to observe, experiment, replicate, and deduce. It demands increasing experimental sophistication as it creates and organizes data into the fabric of truth. In space, life science will require the same dedication and continuity of a large research cadre that has characterized its profound scientific advancements on Earth.

Finally, it is useful to recall that biology in space is no different from that on Earth, because anything living we will carry from Earth into space. Biological science is not looking for new space entities black holes, quasars, galaxies, quarks, and the like - as the physicists are. Biologists will deal with how species that evolved on Earth react to the space environment. By understanding and being able to predict the performance of organisms, tissues, enzymes, and genetic codes in space, biologists can test and refine their science. They can learn about the potential of protoplasm to exist, function, and organize in forms not constrained by gravity, though confined and interdicted by the unique radiation spectrum found there. The living horizon can prove as unlimited as the edge of the universe.
In plotting the future course for NASA's Life Sciences the fundamental scientific questions are preeminent. They deal with (1) how genetic codes turn on and off, (2) how totipotent cells evolve specialized tissue, (3) why cells suddenly revert to totipotency and go out of control, (4) what controls aging, (5) how evolution operates, and (6) why, with current knowledge of genetics, genes, protein biosynthesis, and enzymatic control, has life not yet been created de novo in vitro in the laboratory. There are many others that experience and experiments in space can address from a new vantage point.

The environment in orbits or on long-term interplanetary flights is characterized by:

1. A reduction in the force of gravity to near zero.

2. A space radiation spectrum.

3. A sealed and confined environment.

4. Combinations of all of these.

The task facing the biologist who aspires to contribute to science through space experimentation is to employ these parameters in ways that provide new perspectives to the old problems.

NASA's space science must interact and integrate with the main scientific thrusts of modern biology if it is to bring the unique tools that space affords to answer problems common to all. The new information that will be developed about how organisms cope with space and the new biochemistry revealed will be of immense practical value to those who hope to endure in space, but the scientific target must not be obscured.

In space, as on Earth, the greatest rewards to biological science can be expected to come from understanding, predicting, and ultimately controlling the process and progress of specific genes in organisms and their phenotypic manifestations. The development of such knowledge can be vital to the welfare of humankind in the centuries ahead. It will not be created from studying response to gravity alone. However, no single physical force has been so pervasive, so constant, and so unavoidable as gravity during the millions of years of evolving life in light or darkness, in heat or cold, in water or in air, and at all pressures and elevations. The new opportunities to probe the responses to such a profound determinant of all life on Earth are too precious to ignore any longer. 